\title{
What makes deeply encoded items memorable? Insights into the levels of processing framework from neuroimaging and neuromodulation
}

\section{Giulia Galli * \\ Brain Investigation and Neuromodulation (BIN) Laboratory, Azienda Ospedaliera Universitaria Senese, Siena, Italy}

\section{Edited by: \\ Matteo Feurra, University of Siena Italy}

Reviewed by:

David Bartrés-Faz, University of Barcelona, Spain

Fergus Craik, Baycrest Centre,

Canada

\section{*Correspondence}

Giulia Galli, Brain Investigation and Neuromodulation (BIN) Laboratory Azienda Ospedaliera Universitaria

Senese, Policlinico "Le Scotte," Viale

Bracci 2, Siena 53100, Italy

e-mail: giulgall@gmail.com
When we form new memories, their mnestic fate largely depends upon the cognitive operations set in train during encoding. A typical observation in experimental as well as everyday life settings is that if we learn an item using semantic or "deep" operations, such as attending to its meaning, memory will be better than if we learn the same item using more "shallow" operations, such as attending to its structural features. In the psychological literature, this phenomenon has been conceptualized within the "levels of processing" framework and has been consistently replicated since its original proposal by Craik and Lockhart in 1972. However, the exact mechanisms underlying the memory advantage for deeply encoded items are not yet entirely understood. A cognitive neuroscience perspective can add to this field by clarifying the nature of the processes involved in effective deep and shallow encoding and how they are instantiated in the brain, but so far there has been little work to systematically integrate findings from the literature. This work aims to fill this gap by reviewing, first, some of the key neuroimaging findings on the neural correlates of deep and shallow episodic encoding and second, emerging evidence from studies using neuromodulatory approaches such as psychopharmacology and non-invasive brain stimulation. Taken together, these studies help further our understanding of levels of processing. In addition, by showing that deep encoding can be modulated by acting upon specific brain regions or systems, the reviewed studies pave the way for selective enhancements of episodic encoding processes.

Keywords: levels of processing, episodic memory formation, depth of encoding, TMS, fMRI, tDCS, EEG, psychopharmacology

\section{INTRODUCTION}

Whether we remember an event or not depends on a set of mental processes and brain mechanisms that occur during the initial encoding of the event, its subsequent retrieval, and consolidation processes that take place between encoding and retrieval. Among the factors that act upon encoding, the level to which an item is cognitively processed largely affects memorability. This levels of processing (LOP) framework was originally proposed by Craik and Lockhart in 1972 (1), and has since then fueled debate in episodic memory research. In a typical experiment, depth is manipulated by asking participants to engage deep or shallow processing on the to-be-remembered items during encoding (2). For instance, judging whether a word represents a living or a non-living entity is a deep encoding task because it requires semantic analysis and access to the meaning of the word. By contrast, judging whether a word contains a given letter is a shallow encoding task as it only requires structural and phonological analysis. Other shallow encoding tasks, such as syllable, rhyme, or pleasantness judgments, involve an intermediate level of analysis along the structuralsemantic axis. Typically, items encoded using semantic operations are better remembered in a subsequent memory test than items encoded using shallow operations at any level of depth (2). LOP effects affect later performance even in the absence of any deliberate intention to learn, and are in fact most frequently studied using unintentional encoding. The superiority of memory performance after deep encoding is not only one of the most robust findings in episodic memory research, but it is also clearly recognizable by most experimental participants, and both these factors contribute to the intuitive appeal of the LOP framework.

In general, theorists agree that deep encoding results in more elaborate memory traces, and that this in turn affects later memorability. But what exactly constitutes an elaborate memory trace, and what are the mechanisms that make elaborate memory traces more memorable? The psychological literature has emphasized that enhanced distinctiveness and integration with pre-existing knowledge are among the factors that contribute to the memory benefit for items that received deep processing at encoding $(3,4)$. Yet, it is not entirely clear what are the exact mechanisms underlying LOP effects, and how they are instantiated in the brain. Some authors argue that neuroimaging does not help explain the "experience of memory" and that the debate on LOP effects should remain within the boundaries of experimental psychology (5). However, there are a number of relevant questions that a cognitive neuroscience perspective can help address. For instance, knowing 
which brain regions are activated by deep and shallow encoding and how these activations relate to subsequent memory may inform on the specific processes at play during the two types of encoding, and on the nature of the differences (e.g., qualitative vs. quantitative) between them. Psychopharmacological or noninvasive brain stimulation (NIBS) interventions may add insights into what modulates neural activity associated with deep and shallow encoding in these brain regions, and the relative contribution of encoding and retrieval operations to LOP effects.

This work draws from the cognitive neuroscience literature to describe, first, some of the key neuroimaging findings in this field, and the main questions related to the LOP framework that have been addressed in the past few years of research. I will focus on investigations of neural activity associated with successful episodic memory encoding, therefore on those studies that analyzed neural activity associated with deep and shallow processing at encoding as a function of later memory performance. Clearly, whether a given item will be remembered or not also depends on a set of processes that are specific to the retrieval situation, such as the way memory is probed, and the similarity between encoding and retrieval contexts $(6,7)$. However, an examination of retrievalspecific mechanisms is beyond the aims of the current review, and brain activations associated with retrieval success and recognition memory judgments will not be discussed. I will then review recent findings from CNS-active psychopharmacological interventions that helped clarifying the nature of processes involved in deep and shallow encoding. Finally, I will discuss how NIBS holds promise for future studies aiming at investigating LOP effects, and maybe pave the way for selective enhancements of episodic encoding processes.

\section{NEURAL CORRELATES OF EFFECTIVE DEEP AND SHALLOW ENCODING}

Since the advent of event-related neuroimaging, several studies have investigated the neural correlates of successful memory formation, which involves a comparison of brain activity at encoding, separately for items that are remembered or forgotten in a subsequent memory test. The rationale for this subsequent memory procedure (8) is that determining the neurobiological processes that influence whether an event will be memorable is of vital importance for the understanding of episodic memory. Functional magnetic resonance imaging (fMRI) investigations have consistently reported subsequent memory effects in the ventral and dorsal prefrontal cortex (PFC), medial temporal lobe (MTL), including the hippocampus and parahippocampal regions, and parietal cortex [for reviews see Ref. (9-12)]. In human electrophysiological studies, successful memory formation is indexed by positive-going event-related potentials (ERPs) recorded over anterior scalp sites, and a complex pattern of brain oscillations [for reviews see Ref. $(13,14)$ ]. Only a few studies however (1524) investigated differences in neural activity associated with deep and shallow encoding tasks as a function of subsequent memory performance.

Most of these studies aimed to investigate whether deep and shallow processing leading to successful encoding differ qualitatively or quantitatively. In other words, whether they are expression of distinct mnemonic mechanisms, or, rather, different levels or strengths of a single encoding mechanism. In terms of brain substrates, this translates into the question of whether episodic encoding relies on a single neural system irrespective of encoding task, or it is supported by multiple, task-specific systems. As a general standpoint, this question is complex because it requires a precise separation between deep and shallow encoding, which is in practice hard to achieve. The answer is, indeed, not easy. On the one hand, a good number of studies have demonstrated that a largely similar set of brain regions is implicated in successful deep and shallow encoding $(15,16,19,20)$. More specifically, these studies have shown that the brain regions associated with shallow encoding are a subset of those engaged in deep encoding, with no brain region uniquely associated with the former (15, 16, 19). For instance, Otten et al. (19) demonstrated that remembered words that were deeply studied showed fMRI activations in bilateral inferior frontal gyrus and left anterior and posterior hippocampus, while words encoded in the shallow task elicited subsequent memory effects only in the anterior hippocampus and in a smaller portion of the left inferior frontal gyrus. Evidence of a quantitative, rather than qualitative, difference between deep and shallow subsequent memory effects was also demonstrated in ERPs and magnetoencephalography studies $(21,22,25)$. These findings may suggest that memory formation relies on a single neural system, irrespective of the encoding task.

In contrast with this view, two fMRI studies $(17,24)$ found subsequent memory effects specific for shallow encoding in posterior brain regions, involving the bilateral posterior sulcus, bilateral fusiform gyrus, and left occipital gyrus (17), and an increased functional connectivity between the right hippocampus and the right DLPFC-parietal network (24). However, it should be noted that both Otten and Rugg (17) and Schott et al. (24) used a syllable judgment encoding task (reporting the number of syllables that compose a word). This task, while admittedly shallow, is at an intermediate level of depth compared to the alphabetic task used in the studies reviewed so far. In addition, syllable judgments involve processes that rely on posterior brain regions, such as counting or inferring the number of syllables from the length of the word (26), with only limited engagement of the left PFC (27). Subsequent memory effects in parietal areas associated with a syllable judgment encoding task have indeed been reported before (28). It thus appears that memory formation for syllable judgments involves specific brain regions, which support the online encoding task, whereas other shallow encoding tasks, such as alphabetical judgments, may engage brain activations in prefrontal areas (29), and therefore overlap with those associated with deep encoding. This leads to another relevant question addressed by neuroimaging research, which is central for the understanding of the mechanisms underlying LOP effects, that is, the overlap between task-specific and subsequent memory related activations.

Neuroimaging studies have consistently shown that task- or stimulus-specific brain regions activated during encoding (e.g., areas selectively activated by semantic and structural processing, or by a specific class of stimuli, such as faces) also demonstrated subsequent memory effects $(15-17,19,21,30)$. For instance, the signal increase associated with the deep encoding task in left inferior 
prefrontal and MTL regions mirrored the signal increase that emerged in the subsequent memory contrast for deeply encoded items within the same regions (15-17, 19, 21, 24). Notably, a recent functional connectivity study found increased connectivity between the left PFC and the hippocampus associated with both the semantic task and subsequent memory for deeply encoded stimuli (24). Analogous results in posterior brain regions were demonstrated for shallow encoding $(17,24)$. These findings crucially suggest that memory formation engages the activation of a subset of brain regions that support online, task-specific processing. In other words, effective episodic encoding is supported by products of the processing engaged by the encoding task.

One hypothesis is that during deep encoding, semantic elaboration supported by the left inferior $\operatorname{PFC}(27,31,32)$ automatically activates pre-existing knowledge and semantically associated information about the item, perhaps through a temporary semantic working memory system $(15,17,32)$. The subsequent memory effects in the left inferior PFC and functional connectivity with the hippocampus observed for deep encoding may thus reflect the benefits of incorporating these semantic associations with the studied item into a unique representation of the study episode (10). In other words, during deep encoding items are bound to the contextual aspects of the study episode, which is one of the key components to form a coherent episode in memory (33). It is reasonable to assume that this mechanism is at least in part responsible for the superior memory performance, but also for the higher proportion of confident and recollection-based responses, associated with deep encoding (34). In contrast, shallow encoding tasks that heavily rely on structural processing, such as judging whether a word contains the letter "E," do not engender a sufficiently deep level of analysis to allow associative and contextual processes unfold, and therefore the engagement of relational processes and MTL structures would only be minimal. In between semantic and structural encoding tasks, episodic records for syllable judgments could perhaps incorporate some information derived from the encoding task, such as the word length. This could be reflected in increased functional connectivity between the PFC and the hippocampus for shallow encoding (24).

Taken together, the neuroimaging findings reviewed so far complement and extend previous knowledge on LOP effects, and on episodic memory in general. They suggest that effective deep and shallow encoding may be qualitatively or quantitatively different, depending on the specific processes that are active during the encoding task, and substantiate the idea that the episodic memory of an event is a byproduct of these processes (35). Task-specific and relational processing at encoding, associated with corresponding brain activations, may be ways in which memory formation for deeply encoded items is enhanced.

\section{SELECTIVE MODULATION OF MEMORY FOR DEEPLY ENCODED EVENTS: EVIDENCE FROM PSYCHOPHARMACOLOGICAL STUDIES}

Episodic memory is modulated by a number of neurotransmitters and CNS-active drugs. Studies that investigated the effects of pharmacological interventions on LOP vary with respect to the pharmacokinetic and pharmacodynamic properties of the drug, the dose, and time of administration with respect to the memory phase (encoding or retrieval), and the memory test used to probe memory. That said, there is sufficient commonality in the studies to allow some comparison and integration.

One of the most widely studied neurotransmission systems in relation to memory is the neocortical cholinergic system. Acetylcholine (ACh) projects from the basal forebrain to the cortex and the hippocampus, which contains one of the highest densities of cholinergic terminals and receptors (36). The PFC also shows dense cholinergic innervation (37). Given the key role of these brain structures in learning and memory $(9,10,12)$, the modulation of memory functions by ACh is not surprising. Although the effect of pro-cholinergic drugs is not consistent across studies on healthy young and elderly participants $(38,39)$, acetylcholinesterase inhibitors enhance episodic memory performance in patients with Alzheimer's disease (40), and are routine symptomatic treatments for memory decline in this clinical condition.

A few studies have investigated behavioral and brain activation patterns associated with LOP effects following administration of drugs acting on cholinergic pathways, namely the acetylcholinesterase inhibitors Donepezil and physostigmine $(30,41)$, and nicotine (42). In all these studies, memory accuracy increased following drug administration. In addition, the cholinergic neuromodulation interacted with LOP at encoding, as the memory enhancement was restricted to deeply studied stimuli, while leaving memory accuracy for shallowly encoded items unaffected. This may appear surprising at a first glance - ultimately, deeply encoded items should be less vulnerable to modulations as they involve stronger memory traces. So why would cholinergic effects act upon deeply, but not shallowly, encoded items? A recent fMRI study by Bentley and colleagues (30) offers a plausible explanation. In this study, elderly individuals and Alzheimer's patients received physostigmine or placebo during deep and shallow encoding of images depicting faces or buildings. Volunteers had to judge whether a particular face or building was old or young in the deep encoding task, or whether the image was red or green in the shallow encoding task. For face stimuli, the results showed that in elderly individuals physostigmine increased subsequent memory performance for deep, but not shallowly encoded items. This behavioral advantage was associated with increased activations during deep encoding in the face-selective fusiform cortex, and with increased functional coupling between the fusiform cortex and the right hippocampus. In contrast, in Alzheimer's patients physostigmine did not induce task-dependent behavioral or brain activation changes. These findings substantiate the neuroimaging findings reviewed in the previous section by showing that effective deep encoding is supported by the activation of online, taskor stimulus-specific areas, and by their connections with MTL structures. They further extend previous evidence suggesting that the cholinergic system could be a crucial mediator of this effect. One caveat of the cholinergic studies reviewed so far is that the effect of the drug covered both encoding and retrieval. Given the well-known diverging effects of pro-cholinergic drugs on encoding and retrieval operations (43), future studies could investigate whether the interaction between LOP effects and ACh is dependent upon the time of administration with respect to the memory phase. 
Whereas ACh generally facilitates episodic memory, other neurotransmitter systems are associated with reduced memorability. Ketamine, an antagonist of the $N$-methyl-D-aspartate (NMDA) receptor, and inhibitory neurotransmitters of the gamma-aminobutyric acid (GABA) system, such as benzodiazepines, induce drastic decreases in memory performance (4446). For instance, the facilitation of GABA inhibits the functioning of the hippocampus, inducing dose-related decrements in episodic memory (47). At the neural level, the memory impairment is accompanied by encoding-related deactivations following benzodiazepine administration in the left dorsal PFC (48), left inferior PFC, and hippocampus (49). The modulation of memory performance and brain activations following ketamine and benzodiazepine administration is probably dependent upon the dense concentration of their receptor sites in the hippocampus and cerebral cortex $(50,51)$.

With respect to LOP, the effects of drugs with sedative and amnesic effects have been fairly inconsistent. Lorazepam and ketamine administration was associated with decrements of recognition memory accuracy, selectively for deeply encoded items, or items with an intermediate level of depth (52-54). However, studies from the same groups using similar doses and procedures showed no interaction between drug effects and $\operatorname{LOP}(45,55,56)$. It is not clear how to reconcile these diverging findings. Nevertheless, it is worth noticing that similar to the effects of ACh, the effects of ketamine, and benzodiazepines, if any, act upon deep but not shallow encoding. One could speculate that because of the dense populations of NMDA and benzodiazepine receptors in the frontal cortex and hippocampus, and the extensive recruitment of these brain structures in deep encoding, it is more likely that any disruption would affect deep encoding to a larger extent. Interestingly, Honey et al. (45) demonstrated that following ketamine administration brain activity in the left ventrolateral PFC associated with a deep, compared to a shallow, encoding task increased. This suggests that ketamine may selectively affect task-specific processing that supports successful memory formation. Investigations using other drugs with sedative actions produced additional divergent results, with no interaction between drug and LOP [barbiturates: Ref. (57)], and again selective impairment for deeply encoded items [anesthetic propofol: Ref. $(58,59)$ ].

Finally, and surprisingly given its strong influence on memory (60), cortisol does not seem to interact with LOP. However, the effects of cortisol largely vary depending on dose, timing of administration relative to the memory phase, time of the day of testing, emotional content of the stimulus, and arousal state at the time of testing $(61,62)$. The relation between cortisol and memory is thus very complex, and future studies may find an effect of cortisol on LOP when controlling for some of these variables.

The body of work summarized here suggests that neurotransmitter systems such as cholinergic, GABA-ergic, and NMDA systems have a non-generic sedation or enhancing effect on episodic memory. Perhaps because of their pattern of receptor innervation in the brain, ketamine, ACh, and benzodiazepines selectively affect the memorability of items encoded using deep operations. The modulation of neural activity in brain regions that support the online encoding task may be one way in which CNS-active drugs act upon memory formation of elaborate memory traces. This discussion emphasizes the need of further research on the specific mechanisms that contribute to drug-induced improvements or decrements of episodic memory.

\section{NEUROMODULATION OF DEPTH OF PROCESSING BY NON-INVASIVE BRAIN STIMULATION: EMERGING EVIDENCE}

Functional magnetic resonance imaging and electrophysiological techniques are inherently correlational, therefore, it is not possible on the basis of their data alone to determine whether neural activity is necessary to a specific task. NIBS techniques instead can provide information on the causal role of a specific brain region in a given cognitive process. Transcranial magnetic stimulation (TMS) and transcranial direct current stimulation (tDCS) are the most widely adopted NIBS techniques in the investigation of memory functions.

Transcranial magnetic stimulation uses a magnetic field to induce changes in the resting potentials of the underlying cortex and thus in its electrical currents. This determines a transient interruption of the normal brain activity and interference with cognitive processing (63). TMS can be delivered as a single pulse, or as a series of single pulses (repetitive TMS, rTMS), and can have facilitatory or inhibitory effects depending on the frequency of stimulation. In contrast, tDCS delivers constant, low-intensity (up to $2 \mathrm{~mA}$ ), electrical currents to the scalp via two large anode and cathode electrodes (64). The current modifies resting membrane potentials and the spontaneous firing rate of neurons in a polaritydependent fashion, without however inducing action potentials (65). Because of their distinct physiological mechanisms, TMS and $\mathrm{tDCS}$ differ in the type of information they provide. TMS stimulation is focal, whereas the spatial resolution of tDCS is limited. In addition, TMS is generally locked in time with stimulus presentation, or other events of interest. The temporal dynamics of the engagement of a given brain region can thus be identified by observing the effects of TMS in this region at different points in time (66-69). In contrast, tDCS is not locked to the presentation of single events, rather it is delivered over a prolonged period of time off-line or during the task.

To investigate memory formation, TMS or tDCS is typically delivered over the target area and one or more control sites during encoding, and subsequent memory performance is then assessed as a function of the stimulation condition. On the whole, NIBS studies confirmed previous fMRI and PET evidence of the key role of the PFC in episodic memory formation, either in the dorsal $(68,70-78)$ or ventral $(66,67,79-82)$ portions. It is worth remembering that as the depth of stimulation is limited to a few centimeters, TMS and tDCS cannot directly stimulate some of the key regions involved in episodic memory formation, such as MTL structures. However, a recent neuroimaging study (83) has shown that the stimulation can modulate intrinsic brain network dynamics and propagate to distal brain structures, including the hippocampus.

The majority of brain stimulation studies only adopted one encoding strategy, consisting of a semantic judgment. To date, only two TMS studies directly compared deep and shallow encoding 
tasks and their effect on memory $(76,84)$. Innocenti et al. (76) delivered $10 \mathrm{~Hz}$ rTMS to the left and right dorsolateral PFC during a semantic and an alphabetical judgment encoding task. The effect of the stimulations on subsequent memory performance was compared with the stimulation of a control site and a noTMS condition (baseline). Consistent with previous studies (68, 70-75), rTMS delivered over the left dorsolateral PFC decreased recognition accuracy compared to the other stimulation conditions. However, this effect was specific to semantically encoded words. In the study by Vidal-Piñeiro et al. (84) instead, memory for deep and shallow encoding was equally unaffected by the off-line theta burst stimulation (TBS) of a more ventral region of the PFC. However, as evidenced by a post-TMS fMRI scan, TBS increased activations of the left ventrolateral PFC, occipital cortex, and cerebellum, and the connectivity between these brain regions, while volunteers were performing the deep encoding task. These findings suggest that the combination of neuroimaging and brain stimulation offers relevant insights into the brain networks involved in LOP effects, even in the absence of overt behavioral effects.

There are several methodological differences between these two TMS studies that may have determined the discrepancy of behavioral effects, including differences in the protocol and site of stimulation. Nevertheless, once again the literature offers a scenario in which the neural or behavioral modulation is specific to semantic encoding. Along the same vein of what has been suggested for psychopharmacological studies, neuromodulation with TMS may interfere with task-specific and associative processes that support the online semantic task. Unfortunately, performance for the semantic encoding task is generally at ceiling, and this makes it hard to detect any effect of neuromodulation at encoding. In fact, investigations that used a neuromodulatory approach either did not report performance data for the encoding tasks, or reported a lack of effects.

In summary, TMS holds promise for future investigations of LOP effects. The possibility to selectively interfere with specific stages of memory (encoding vs. retrieval) makes this technique an excellent candidate for the study of the relative role of encoding and retrieval operations in determining LOP effects. Further studies using different stimulation protocols, sites, and timings are needed to expand our knowledge of selective effects of TMS on deep encoding. Electrical stimulation with tDCS could also further our understanding of LOP effects. For instance, the differential effects of anodal and cathodal tDCS on episodic memory performance (85) may induce dissociations in LOP effects, thereby adding to the investigation of the nature of the differences between deep and shallow encoding. In addition, given that anodal tDCS induces enhancements in episodic memory performance in healthy young and elderly individuals (78), it will be of great interest in future studies to assess whether tDCS can selectively induce memory enhancements according to the depth of encoding. This could be relevant especially for those pathological conditions that are characterized by memory impairments of deep but not shallow encoding (86-88). Finally, the observation that subsequent memory for deep and shallow encoding is associated with different patterns of oscillatory brain activity $(23,89,90)$, will provide impetus for the investigation of the effects of rhythmic brain stimulation (rTMS and transcranial alternating current stimulation) on depth of processing.

\section{CONCLUSION}

In this article, I reviewed the contribution of neuroimaging, psychopharmacological, and NIBS studies to our understanding of LOP effects. Taken together, the findings discussed here provide partial answers to the question of "what makes deeply encoded items more memorable?" They suggest that memory formation for deeply encoded events is enhanced when the products of online, task-specific processing are integrated with pre-existing knowledge about the event into a coherent episodic memory trace. At the neural level, this is reflected in overlapping task- and encoding-related brain activations, and their functional connections with MTL structures. These findings therefore converge with the psychological literature, which has previously suggested that the episodic memory of an event is a byproduct of the processes active during encoding (35), and that the integration with preexisting knowledge contributes to the memory benefit for items that receive deep processing at encoding $(3,4)$. Crucially, these cognitive and neural processes are mediated by activity in cholinergic, GABA-ergic, and NMDA neurotransmitter systems, which analogously to NIBS, specifically modulate memory formation for deep encoding. The proposed mechanism may not be exclusive to deep encoding per se. Rather, it may generalize to shallow encoding task that are of sufficient depth to induce associative process in the formation of the episodic record.

It is important to note that a process-based account need not be the only explanation for the specificity of the effects for deep encoding. For instance, the number of trials for shallow encoding in any given subsequent memory comparison is generally small due to low memory performance. Therefore, the power to detect any statistical difference in this condition is low. One could speculate that distinct subsequent memory patterns would emerge if a shallow encoding task that yields higher memory accuracy was used. In this view, the posterior subsequent memory effects for shallow encoding reported in Otten and Rugg (17) and Schott et al. (24) could be attributable to the higher number of trials in the syllable judgment encoding task, rather than to the specific processes involved in this task. Distinguishing between these alternative views will be difficult, but future studies could address this issue through careful examinations of how systematic variations of encoding tasks yielding different levels of memory accuracy correspond to linear changes in brain activation patterns.

Finally, one should emphasize that the mechanisms enhancing memory formation for deeply encoded events reviewed here provide only part of what is needed to accurately remember those events, that is, they provide the potential of retrieval (4). Equally important are the processes that occur during retrieval, the way memory is later tested, and the overlap of the encoding and retrieval situation $(6,7)$. The possibility to selectively interfere with different stages of the memory process make neuromodulatory approaches excellent candidates for the investigation of the interdependence of encoding and retrieval operations. 


\section{REFERENCES}

1. Craik FIM, Lockhart RS. Levels of processing: a framework for memory research. J Verbal Learning Verbal Behav (1972) 11:671-84. doi:10.1016/S0022-5371(72) 80001-X

2. Craik FIM, Tulving E. Depth of processing and the retention of words in episodic memory. J Exp Psychol Gen (1975) 104:268-94. doi:10.1037/0096-3445.104.3. 268

3. Craik FIM. Levels of encoding and retrieval. In: Challis BH, Velichkovskii BH, editors. Stratification in Cognition and Consciousness. Amsterdam: John Benjamins (1999). p. 97-104.

4. Craik FIM. Levels of processing: past, present, and future? Memory (2002) 10:305-18. doi:10.1080/09658210244000135

5. Watkins MJ. Limits and province of levels of processing: considerations of a construct. Memory (2002) 10:339-43. doi:10.1080/09658210244000162

6. Tulving E, Thompson D. Encoding specificity and retrieval processes in episodic memory. Psychol Rev (1973) 80:352-73. doi:10.1037/h0020071

7. Morris CD, Bransford JD, Franks JJ. Levels of processing versus transfer appropriate processing. J Verbal Learning Verbal Behav (1977) 16:519-33. doi:10.1080/09658210903266931

8. Sanquist TF, Rohrbaugh JW, Syndulko K, Lindsley DB. Electrocortical signs of levels of processing: perceptual analysis and recognition memory. Psychophysi$\operatorname{olog} y$ (1980) 17:568-76. doi:10.1111/j.1469-8986.1980.tb02299.x

9. Paller KA, Wagner AD. Observing the transformation of experience into memory. Trends Cogn Sci (2002) 6:93-102. doi:10.1016/S1364-6613(00)01845-3

10. Davachi L. Item, context and relational episodic encoding in humans. Curr Opin Neurobiol (2006) 16:693-700. doi:10.1016/j.conb.2006.10.012

11. Uncapher MR, Wagner AD. Posterior parietal cortex and episodic encoding: insights from fMRI subsequent memory effects and dual-attention theory. Neurobiol Learn Mem (2009) 9:139-54. doi:10.1016/j.nlm.2008.10.011

12. Ranganath CA. Unified framework for the functional organization of the medial temporal lobes and the phenomenology of episodic memory. Hippocampus (2010) 20:1263-90. doi:10.1002/hipo.20852

13. Friedman D, Johnson R. Event-related potential (ERP) studies of memory encoding and retrieval: a selective review. Microsc Res Tech (2000) 51:6-28. doi:10.1002/1097-0029(20001001)51:1<6::AID-JEMT2>3.3.CO;2-I

14. Nyhus E, Curran T. Functional role of gamma and theta oscillations in episodic memory. Neurosci Biobehav Rev (2010) 34:1023-35. doi:10.1016/j.neubiorev. 2009.12.014

15. Wagner AD, Schacter DL, Rotte M, Koutstaal W, Maril A, Dale AM, et al. Building memories: remembering and forgetting of verbal experiences as predicted by brain activity. Science (1998) 21:1188-91. doi:10.1126/science.281. 5380.1188

16. Baker JT, Sanders AL, Maccotta L, Buckner RL. Neural correlates of verbal memory encoding during semantic and structural processing tasks. Neuroreport (2001) 12:1251-6. doi:10.1097/00001756-200105080-00039

17. Otten LJ, Rugg MD. Task-dependency of the neural correlates of episodic encoding as measured by fMRI. Cereb Cortex (2001) 11:1150-60. doi:10.1093/cercor/ 11.12.1150

18. Otten LJ, Rugg MD. Electrophysiological correlates of memory encoding are task-dependent. Brain Res Cogn Brain Res (2001) 12:11-8. doi:10.1016/S09266410(01)00015-5

19. Otten LJ, Henson RN, Rugg MD. Depth of processing effects on neural correlates of memory encoding: relationship between findings from across- and withintask comparisons. Brain (2001) 124:399-412. doi:10.1093/brain/124.2.399

20. Fletcher PC, Stephenson CM, Carpenter TA, Donovan T, Bullmore ET. Regional brain activations predicting subsequent memory success: an event-related fMRI study of the influence of encoding tasks. Cortex (2003) 39:1009-26. doi:10.1016/S0010-9452(08)70875-X

21. Staresina BP, Bauer H, Deecke L, Walla P. Neurocognitive correlates of incidental verbal memory encoding: a magnetoencephalographic (MEG) study. Neuroimage (2005) 25:430-43. doi:10.1016/j.neuroimage.2004.11.035

22. Guderian S, Schott BH, Richardson-Klavehn A, Düzel E. Medial temporal theta state before an event predicts episodic encoding success in humans. Proc Natl Acad Sci U S A (2009) 106:5365-70. doi:10.1073/pnas.0900289106

23. Hanslmayr S, Spitzer B, Bäuml KH. Brain oscillations dissociate between semantic and nonsemantic encoding of episodic memories. Cereb Cortex (2009) 19:1631-40. doi:10.1093/cercor/bhn197
24. Schott BH, Wüstenberg T, Wimber M, Fenker DB, Zierhut KC, Seidenbecher $\mathrm{CI}$, et al. The relationship between level of processing and hippocampal-cortical functional connectivity during episodic memory formation in humans. Hum Brain Mapp (2013) 34:407-24. doi:10.1002/hbm.21435

25. Friedman D, Ritter W, Snodgrass JG. ERPs during study as a function of subsequent direct and indirect memory testing in young and old adults. Brain Res Cogn Brain Res (1996) 4:1-13. doi:10.1016/0926-6410(95)00041-0

26. Paulesu E, Frith CD, Frackowiak RS. The neural correlates of the verbal component of working memory. Nature (1993) 362:342-5. doi:10.1038/362342a0

27. Poldrack RA, Wagner AD, Prull MW, Desmond JE, Glover GH, Gabrieli JD. Functional specialization for semantic and phonological processing in the left inferior prefrontal cortex. Neuroimage (1999) 10:15-35. doi:10.1006/nimg.1999. 0441

28. Clark D, Wagner AD. Assembling and encoding word representations: fMRI subsequent memory effects implicate a role for phonological control. Neuropsychologia (2003) 41:304-17. doi:10.1016/S0028-3932(02)00163-X

29. Gold BT, Buckner RL. Common prefrontal regions coactivate with dissociable posterior regions during controlled semantic and phonological tasks. Neuron (2002) 15:803-12. doi:10.1016/S0896-6273(02)00800-0

30. Bentley P, Driver J, Dolan RJ. Modulation of fusiform cortex activity by cholinesterase inhibition predicts effects on subsequent memory. Brain (2009) 132:2356-71. doi:10.1093/brain/awp176

31. Kapur S, Craik FIM, Tulving E, Wilson AA, Houle S, Brown GM. Neuroanatomical correlates of encoding in episodic memory: levels of processing effect. Proc Natl Acad Sci U S A (1994) 91:2008-11. doi:10.1073/pnas.91.6.2008

32. Gabrieli JD, Poldrack RA, Desmond JE. The role of left prefrontal cortex in language and memory. Proc Natl Acad Sci U S A (1998) 95:906-13. doi:10.1073/pnas.95.3.906

33. Tulving E. Episodic and semantic memory. In: Tulving E, Donaldson W, editors. Organization of Memory. New York, NY: Academic Press (1972). p. 381-403.

34. Gardiner TM, Richardson-Klavehn A. Remembering and knowing. In: Tulving E, Craik FIM, editors. The Oxford Handbook of Memory. New York, NY: Oxford University Press (2000). p. 229-44.

35. Lockhart RS. Levels of processing. In: Squire LR, editor. Encyclopedia of Learning and Memory. New York, NY: Macmillan (1992). p. 106-8.

36. Mesulam MM, Volicer L, Marquis JK, Mufson EJ, Green RC. Systematic regional differences in the cholinergic innervation of the primate cerebral cortex: distribution of enzyme activities and some behavioral implications. Ann Neurol (1986) 19:144-51. doi:10.1002/ana.410190206

37. Wallace TL, Bertrand D. Importance of the nicotinic acetylcholine receptor system in the prefrontal cortex. Biochem Pharmacol (2013) 85:1713-20. doi:10.1016/j.bcp.2013.04.001

38. Repantis D, Laisney O, Heuser I. Acetylcholinesterase inhibitors and memantine for neuroenhancement in healthy individuals: a systematic review. Pharmacol Res (2010) 61:473-81. doi:10.1016/j.phrs.2010.02.009

39. Balsters JH, O'Connell RG, Martin MP, Galli A, Cassidy SM, Kilcullen SM, et al. Donepezil impairs memory in healthy older subjects: behavioural, EEG and simultaneous EEG/fMRI biomarkers. PLoS One (2011) 6(9):e24126. doi: 10.1371/journal.pone.0024126

40. Rogers SL, Farlow MR, Doody RS, Mohs R, Friedhoff LT. A 24-week, doubleblind, placebo-controlled trial of donepezil in patients with Alzheimer's disease. Donepezil Study Group. Neurology (1998) 50:136-45. doi:10.1212/WNL.50.1. 136

41. FitzGerald DB, Crucian GP, Mielke JB, Shenal BV, Burks D, Womack KB, et al. Effects of donepezil on verbal memory after semantic processing in healthy older adults. Cogn Behav Neurol (2008) 21:57-64. doi:10.1097/WNN. 0b013e3181799df1

42. Warburton DM, Skinner A, Martin CD. Improved incidental memory with nicotine after semantic processing, but not after phonological processing. Psychopharmacology (2001) 153:258-63. doi:10.1007/s002130000565

43. Hasselmo ME. Neuromodulation and cortical function: modeling the physiological basis of behavior. Behav Brain Res (1995) 67:1-2. doi:10.1016/01664328(94)00113-T

44. Hetem LA, Danion JM, Diemunsch P, Brandt C. Effect of a subanesthetic dose of ketamine on memory and conscious awareness in healthy volunteers. Psychopharmacology (2000) 152:283-8. doi:10.1007/s002130000511 
45. Honey GD, Honey RA, O’Loughlin C, Sharar SR, Kumaran D, Suckling J, et al. Ketamine disrupts frontal and hippocampal contribution to encoding and retrieval of episodic memory: an fMRI study. Cereb Cortex (2005) 15:749-59. doi:10.1093/cercor/bhh176

46. Morgan CJ, Curran HV. Acute and chronic effects of ketamine upon human memory: a review. Psychopharmacology (2006) 188:408-24. doi:10.1007/ s00213-006-0572-3

47. Curran HV. Benzodiazepines, memory and mood: a review. Psychopharmacology (1991) 105:1-8. doi:10.1007/BF02316856

48. Coull JT, Frith CD, Dolan RJ. Dissociating neuromodulatory effects of diazepam on episodic memory encoding and executive function. Psychopharmacology (1999) 145:213-22. doi:10.1007/s002130051051

49. Sperling R, Greve D, Dale A, Killiany R, Holmes J, Rosas HD, et al. Functional MRI detection of pharmacologically induced memory impairment. Proc Natl Acad Sci U S A (2002) 99:455-60. doi:10.1073/pnas.012467899

50. Mohler H, Richards JG. Receptors for anxiolytic drugs. In: Mallick JB, Enna SJ, Yamamura HI, editors. Anxiolytics: Neurochemical, Behavioral, and Clinical Perspectives. New York, NY: Raven Press (1983). p. 15-40.

51. Newcomer JW, Krystal JH. NMDA receptor regulation of memory and behavior in humans. Hippocampus (2001) 11:529-42. doi:10.1002/hipo.1069

52. Bishop KI, Curran HV. Psychopharmacological analysis of implicit and explicit memory: a study with lorazepam and the benzodiazepine antagonist flumazenil. Psychopharmacology (1995) 121:267-78. doi:10.1007/BF02245638

53. Honey GD, Honey RA, Sharar SR, Turner DC, Pomarol-Clotet E, Kumaran $\mathrm{D}$, et al. Impairment of specific episodic memory processes by sub-psychotic doses of ketamine: the effects of levels of processing at encoding and of the subsequent retrieval task. Psychopharmacology (2005) 181:445-57. doi:10.1007/ s00213-005-0001-z

54. Honey GD, O'loughlin C, Turner DC, Pomarol-Clotet E, Corlett PR, Fletcher PC. The effects of a subpsychotic dose of ketamine on recognition and source memory for agency: implications for pharmacological modelling of core symptoms of schizophrenia. Neuropsychopharmacology (2006) 31:413-23. doi:10.1038/sj.npp.1300846

55. Curran HV, Barrow S, Weingartner H, Lader M, Bernik M. Encoding, remembering and awareness in Lorazepam-induced amnesia. Psychopharmacology (1995) 122:187-93. doi:10.1007/BF02246094

56. Morgan CJ, Mofeez A, Brandner B, Bromley L, Curran HV. Acute effects of ketamine on memory systems and psychotic symptoms in healthy volunteers. Neuropsychopharmacology (2004) 29:208-18. doi:10.1038/sj.npp. 1300342

57. Williams HL, Rundell OH. Secobarbital effects on recall and recognition in a levels-of-processing paradigm. Psychopharmacology (1983) 80:221-225. doi:10.1007/BF00436157

58. N'Kaoua B, Véron AL, Lespinet VC, Claverie B, Sztark F. Time course of cognitive recovery after propofol anaesthesia: a level of processing approach. J Clin Exp Neuropsychol (2002) 24:713-9. doi:10.1076/jcen.24.6.713.8401

59. Veselis RA, Pryor KO, Reinsel RA, Mehta M, Pan H, Johnson R. Low-dose propofol-induced amnesia is not due to a failure of encoding: left inferior prefrontal cortex is still active. Anesthesiology (2008) 109:213-24. doi:10.1097/ALN. 0b013e31817fd8ae

60. de Quervain DJ, Roozendaal B, Nitsch RM, McGaugh JL, Hock C. Acute cortisone administration impairs retrieval of long-term declarative memory in humans. Nat Neurosci (2000) 3:313-4. doi:10.1038/73873

61. Domes G, Rothfischer J, Reichwald U, Hautzinger M. Inverted-U function between salivary cortisol and retrieval of verbal memory after hydrocortisone treatment. Behav Neurosci (2005) 119:512-7. doi:10.1037/0735-7044.119.2.512

62. Sandi C. Glucocorticoids act on glutamatergic pathways to affect memory processes. Trends Neurosci (2011) 34:165-76. doi:10.1016/j.tins.2011.01.006

63. Walsh V, Cowey A. Transcranial magnetic stimulation and cognitive neuroscience. Nat Rev Neurosci (2000) 1:73-9. doi:10.1038/35036239

64. Nitsche MA, Paulus W. Excitability changes induced in the human motor cortex by weak transcranial direct current stimulation. J Physiol (2000) 527:633-9. doi:10.1111/j.1469-7793.2000.t01-1-00633.x

65. Zaghi S, Acar M, Hultgren B, Boggio PS, Fregni F. Noninvasive brain stimulation with low-intensity electrical currents: putative mechanisms of action for direct and alternating current stimulation. Neuroscientist (2010) 16:285-307. doi: $10.1177 / 1073858409336227$
66. Kahn I, Pascual-Leone A, Theoret H, Fregni F, Clark D, Wagner AD. Transient disruption of ventrolateral prefrontal cortex during verbal encoding affects subsequent memory performance. J Neurophysiol (2005) 94:688-98. doi:10.1152/jn.01335.2004

67. Machizawa MG, Kalla R, Walsh V, Otten LJ. The time course of ventrolateral prefrontal cortex involvement in memory formation. J Neurophysiol (2010) 103:1569-79. doi:10.1152/jn.90937.2008

68. Rossi S, Innocenti I, Polizzotto NR, Feurra M, De Capua A, Ulivelli M, et al. Temporal dynamics of memory trace formation in the human prefrontal cortex. Cereb Cortex (2011) 21:368-73. doi:10.1093/cercor/bhq103

69. Turriziani P, Oliveri M, Salerno S, Costanzo F, Koch G, Caltagirone C, et al. Recognition memory and prefrontal cortex: dissociating recollection and familiarity processes using rTMS. Behav Neurol (2008) 19:23-7. doi:10.1155/2008/ 568057

70. Rossi S, Cappa SF, Babiloni C, Pasqualetti P, Miniussi C, Carducci F, et al. Prefrontal cortex in long-term memory: an "interference" approach using magnetic stimulation. Nat Neurosci (2001) 4:948-52. doi:10.1038/nn0901-948

71. Rossi S, Miniussi C, Pasqualetti P, Babiloni C, Rossini PM, Cappa SF. Agerelated functional changes of prefrontal cortex in long-term memory: a repetitive transcranial magnetic stimulation study. J Neurosci (2004) 24:7939-44. doi:10.1523/JNEUROSCI.0703-04.2004

72. Rossi S, Pasqualetti P, Zito G, Vecchio F, Cappa SF, Miniussi C, et al. Prefrontal and parietal cortex in human episodic memory: an interference study by repetitive transcranial magnetic stimulation. Eur J Neurosci (2006) 23:793-800. doi:10.1111/j.1460-9568.2006.04600.x

73. Sandrini M, Cappa SF, Rossi S, Rossini PM, Miniussi C. The role of prefrontal cortex in verbal episodic memory: rTMS evidence. J Cogn Neurosci (2003) 15:855-61. doi:10.1162/089892903322370771

74. Skrdlantová L, Horácek J, Dockery C, Lukavský J, Kopecek M, Preiss M, et al. The influence of low-frequency left prefrontal repetitive transcranial magnetic stimulation on memory for words but not for faces. Physiol Res (2005) 54: 123-8.

75. Gagnon G, Blanchet S, Grondin S, Schneider C. Paired-pulse transcranial magnetic stimulation over the dorsolateral prefrontal cortex interferes with episodic encoding and retrieval for both verbal and non-verbal materials. Brain Res (2010) 1344:148-58. doi:10.1016/j.brainres.2010.04.041

76. Innocenti I, Giovannelli F, Cincotta M, Feurra M, Polizzotto NR, Bianco G, et al. Event-related rTMS at encoding affects differently deep and shallow memory traces. Neuroimage (2010) 53:325-30. doi:10.1016/j.neuroimage.2010. 06.011

77. Javadi AH, Walsh V. Transcranial direct current stimulation (tDCS) of the left dorsolateral prefrontal cortex modulates declarative memory. Brain Stimul (2012) 5:231-41. doi:10.1016/j.brs.2011.06.007

78. Manenti R, Brambilla M, Petesi M, Ferrari C, Cotelli M. Enhancing verbal episodic memory in older and young subjects after non-invasive brain stimulation. Front Aging Neurosci (2013) 5:49. doi:10.3389/fnagi.2013.00049

79. Köhler S, Paus T, Buckner RL, Milner B. Effects of left inferior prefrontal stimulation on episodic memory formation: a two-stage fMRI-rTMS study. J Cogn Neurosci (2004) 16:178-88. doi:10.1162/089892904322984490

80. Floel A, Poeppel D, Buffalo EA, Braun A, Wu CW, Seo HJ, et al. Prefrontal cortex asymmetry for memory encoding of words and abstract shapes. Cereb Cortex (2004) 14:404-9. doi:10.1093/cercor/bhh002

81. Feurra M, Fuggetta G, Rossi S, Walsh V. The role of the left inferior frontal gyrus in episodic encoding of faces: an interference study by repetitive transcranial magnetic stimulation. Cogn Neurosci (2010) 1:118-25. doi:10.1080/ 17588921003660736

82. Blumenfeld RS, Lee TG, D'Esposito M. The effects of lateral prefrontal transcranial magnetic stimulation on item memory encoding. Neuropsychologia (2014) 53:197-202. doi:10.1016/j.neuropsychologia.2013.11.021

83. Eldaief MC, Halko MA, Buckner RL, Pascual-Leone A. Transcranial magnetic stimulation modulates the brain's intrinsic activity in a frequency-dependent manner. Proc Natl Acad Sci U S A (2011) 108:21129-34. doi:10.1073/pnas. 1113103109

84. Vidal-Piñeiro D, Martin-Trias P, Arenaza-Urquijo EM, Sala-Llonch R, Clemente IC, Mena-Sánchez I, et al. Task-dependent activity and connectivity predict episodic memory network-based responses to brain stimulation in healthy aging. Brain Stimul (2014) 7:287-96. doi:10.1016/j.brs.2013.12.016 
85. Brasil-Neto JP. Learning, memory, and transcranial direct current stimulation. Front Psychiatry (2012) 3:80. doi:10.3389/fpsyt.2012.00080

86. Toichi M, Kamio Y. Long-term memory and levels-of-processing in autism. Neuropsychologia (2002) 40:964-9. doi:10.1016/S0028-3932(01)00163-4

87. Bonner-Jackson A, Haut KM, Csernansky JG, Barch DM. The influence of encoding strategy on episodic memory and cortical activity in schizophrenia. Biol Psychiatry (2005) 58:47-55. doi:10.1016/j.biopsych.2005.05.011

88. Mandzia JL, McAndrews MP, Grady CL, Graham SJ, Black SE. Neural correlates of incidental memory in mild cognitive impairment: an fMRI study. Neurobiol Aging (2009) 30:717-30. doi:10.1016/j.neurobiolaging.2007.08.024

89. Hanslmayr S, Volberg G, Wimber M, Raabe M, Greenlee MW, Bäuml KH. The relationship between brain oscillations and BOLD signal during memory formation: a combined EEG-fMRI study. J Neurosci (2011) 31:15674-80. doi:10.1523/JNEUROSCI.3140-11.2011

90. Hanslmayr S, Staudigl T. How brain oscillations form memories - a processing based perspective on oscillatory subsequent memory effects. Neuroimage (2014) 85:648-55. doi:10.1016/j.neuroimage.2013.05.121
Conflict of Interest Statement: The author declares that the research was conducted in the absence of any commercial or financial relationships that could be construed as a potential conflict of interest.

Received: 01 April 2014; paper pending published: 22 April 2014; accepted: 15 May 2014; published online: 28 May 2014.

Citation: Galli $G$ (2014) What makes deeply encoded items memorable? Insights into the levels of processing framework from neuroimaging and neuromodulation. Front. Psychiatry 5:61. doi: 10.3389/fpsyt.2014.00061

This article was submitted to Neuropsychiatric Imaging and Stimulation, a section of the journal Frontiers in Psychiatry.

Copyright $(2014$ Galli. This is an open-access article distributed under the terms of the Creative Commons Attribution License (CC BY). The use, distribution or reproduction in other forums is permitted, provided the original author(s) or licensor are credited and that the original publication in this journal is cited, in accordance with accepted academic practice. No use, distribution or reproduction is permitted which does not comply with these terms. 